\title{
SKEW-PRODUCT FLOWS, FINITE EXTENSIONS OF MINIMAL TRANSFORMATION GROUPS AND ALMOST PERIODIC DIFFERENTIAL EQUATIONS ${ }^{1}$
}

\author{
BY ROBERT J. SACKER ${ }^{2}$ AND GEORGE R. SELL ${ }^{3}$ \\ Communicated by Walter Gottschalk, January 11, 1973
}

I. Skew-product flows. A flow $\pi$ on a product space $X \times Y$ is said to be a skew-product flow if there exist continuous mappings $\varphi: X \times Y \times T$ $\rightarrow X$ and $\sigma: Y \times T \rightarrow Y$ such that

$$
\pi(x, y, t)=(\varphi(x, y, t), \sigma(y, t))
$$

where $\sigma$ is itself a flow on $Y$ and $T$ is a topological group. In other words the natural projection $p: X \times Y \rightarrow Y$ is a homomorphism of the transformation group $(X \times Y, T, \pi)$ onto $(Y, T, \sigma)$.

Skew-product flows arise in a natural way in the study of ordinary differential equations $x^{\prime}=g(x, t)$ (cf. [6] and [7]). In this case the group $T$ would be the real numbers and $Y$ would be a topological function space containing $g$ and closed under time-translations. The flow $\sigma$ would be given by $\sigma(f, \tau)=f_{\tau}$ where $f_{\tau}(x, t)=f(x, \tau+t)$. The space $X$ would be the phase space for the differential equation, usually $X$ is the Euclidean space $R^{n}$ or perhaps some $n$-dimensional manifold, and $\varphi(x, f, t)$ would represent the solution of $x^{\prime}=f(x, t)$ passing through $x$ at time $t=0$. (We assume that all differential equations in $Y$ give rise to unique solutions, although some of our results are valid without this restriction (cf. [8]).)

Now assume that $Y$ is a compact minimal set under the flow $\sigma$ and let $M \subset X \times Y$ be a compact invariant set of the skew-product flow. Motivated by the above model for differential equations we ask: When can certain structures be lifted from $Y$ to $M$ ? For example, if we assume that $Y$ is an almost periodic minimal set (that is, the flow $\sigma$ is equicontinuous on $Y$ ) under what conditions will $M$ contain an almost periodic minimal set?

We shall say that the flow $\pi$ has the distal property on $M$ if for any $y \in Y$ and $x_{1}, x_{2} \in X$ with $x_{1} \neq x_{2},\left(x_{1}, y\right) \in M$ and $\left(x_{2}, y\right) \in M$ there is an

AMS (MOS) subject classifications (1970). Primary 22A99, 34C25, 34C35, 34C40, 54H20.

Key words and phrases. Almost periodic differential equation, almost periodic solution, covering space, distal, equicontinuous, skew-product flow, transformation group.

${ }^{1}$ This research was begun while visiting at the Istituto di Matematica dell' Università di Firenze under the auspices of the Italian Research Council (C.N.R.).

${ }^{2}$ Partially supported by U.S. Army Grant DA-ARO-D-31-124-71-G176.

${ }^{3}$ Partially supported by NSF Grant No. GP-27275. 
$\alpha=\alpha\left(x_{1}, x_{2}, y\right)>0$ such that $d\left(\varphi\left(x_{1}, y, t\right), \varphi\left(x_{2}, y, t\right)\right) \geqq \alpha$ for all $t \in R^{+}$. Here $d$ denotes a metric on $X$. (For our purposes the $R^{+}$above may be replaced by $R^{-}$.)

We can now prove the following theorem [4], [5]:

THeOREM 1. Assume that $Y$ is a compact uniform Hausdorff space and the flow $\sigma$ is minimal on $Y$. Assume that $X$ is metrizable and $T=R$. Let $M \subset X \times Y$ be a compact invariant set for the flow $\pi$ and assume either:

(I) $\operatorname{card}\left(p^{-1}(y) \cap M\right)=N<\infty$ for all $y \in Y$, where $N$ does not depend on $y$, or

(II) $\operatorname{card}\left(p^{-1}\left(y_{0}\right) \cap M\right)=N<\infty$ for some $y_{0} \in Y$ and $\pi$ has the distal property on $M$.

Then $M$ is an $N$-fold covering space of $Y$. Also $M$ can be written as the finite union of minimal sets. If, in addition, $Y$ is almost periodic minimal then every minimal set in $M$ is also almost periodic.

The assumption that $X$ be metrizable (and not merely a uniform space) is used in a crucial way in our proof. The fact that $Y$ can be a nonmetrizable uniform space does arise in differential equations when $Y$ has a weak topology. In the case that both $X$ and $Y$ are metrizable then Theorem 1 is a consequence of a more general result which we now describe.

II. Finite extensions of minimal transformation groups. Recall that a continuous mapping $p$ of a transformation group $(W, T, \pi)$ onto a transformation group $(Y, T, \sigma)$ is said to be a homomorphism if $p$ commutes with $t$, that is, if $\sigma(p(w), t)=p(\pi(w, t))$. Also $p$ is said to be a homomorphism of distal type if whenever $w_{1}, w_{2} \in p^{-1}(y)$ with $w_{1} \neq w_{2}$, there is an $\alpha=\alpha\left(w_{1}, w_{2}\right)>0$ such that $d\left(\pi\left(w_{1}, t\right), \pi\left(w_{2}, t\right)\right) \geqq \alpha$ for all $t \in T$. The space $W$ is said to be a finite $\left(N\right.$-to-1) extension of $Y$ if $\operatorname{card} p^{-1}(y)=N<\infty$ for all $y \in Y$.

The next result places no restriction on the topological group $T$.

THEOREM 2. Let $W$ and $Y$ be compact metric spaces where the flow $\sigma$ on $Y$ is minimal. Let $p: W \rightarrow Y$ be a homomorphism. Then the following statements are equivalent:

(I) $W$ is a finite ( $N$-to-1) extension of $Y$.

(II) $p$ is of distal type and card $p^{-1}\left(y_{0}\right)=N$ for some $y_{0} \in Y$.

(III) $W$ is an $N$-fold covering space of $Y$ with covering projection $p$.

In $[2$, p. 56], R. Ellis asks whether an equicontinuous structure on $Y$ can be lifted to a finite ( $N$-to-1) extension of $Y$. We can give an affirmative answer, but now we must place a rather mild restriction on the group $T$.

THEOREM 3. Let $p: W \rightarrow Y$ be a homomorphism where $W$ and $Y$ are compact metric spaces. Assume the following: 
(I) $(Y, T, \sigma)$ is equicontinuous.

(II) $W$ is an $N$-fold covering space of $Y$ with covering projection $p$.

(III) The group $T$ has the property that there is a compact subset $K \subset T$ such that $T$ is generated by any open neighborhood of $K$.

Then $(W, T, \pi)$ is equicontinuous.

The class $\mathscr{T}$ of topological groups that satisfy condition (III) above is very large. $\mathscr{T}$ contains all compactly generated groups, all connected groups, and $\mathscr{T}$ is closed under arbitrary products with the standard product topology. However, $\mathscr{T}$ does not include infinitely generated discrete groups.

III. Almost periodic differential equations. Let us now return to the differential equation model described in $\S I$, where we now assume that $Y$ is an almost periodic minimal set. This means that $Y$ is the hull $H(g)$ generated by a differential equation $x^{\prime}=g(x, t)$ where $g$ is uniformly Bohr almost periodic in $t$ (cf. [7]). The problem of determining whether a set $M \subset X \times Y$ contains an almost periodic minimal set is the same as asking whether the given differential equation $x^{\prime}=g(x, t)$ has an almost periodic solution (cf. [7]). If $x^{\prime}=g(x, t)$ has a positively compact solution $\varphi(x, g, t)$, that is, $\varphi$ remains in a compact set for $t \geqq 0$, then the $\omega$-limit set $M=\Omega_{(x, g)}$ is a compact invariant set in $X \times Y$. If the positively compact solution $\varphi(x, g, t)$ is uniformly stable [7] then we can show that the solutions have the distal property on $M$, and that $M$ is a minimal set. For an application of Theorem 1, it remains only to check the finiteness condition card $\left(p^{-1}\left(y_{0}\right) \cap M\right)=N<\infty$ for some $y_{0} \in Y$. However, if the positively compact solution $\varphi(x, g, t)$ is uniformly asymptotically stable then we can verify this finiteness condition; and hence $M$ is an $N$-fold covering of $Y$ and there exists an almost periodic solution of $x^{\prime}=g(x, t)$. Thus the theorems of R. K. Miller [3] and T. Yoshizawa [9] are special cases of Theorem 1.

The theory of L. Amerio [1] is also included in Theorem 1. He assumed a separatedness condition which is much stronger than the distal property used in Theorem 1. This separatedness condition already implies the finiteness condition card $\left(p^{-1}\left(y_{0}\right) \cap M\right)=N<\infty$.

For the scalar-valued differential equation $x^{\prime}=g(x, t)$ we can prove the following result.

THEOREM 4. Let $x^{\prime}=g(x, t)$ be a scalar-valued differential equation where $g$ is uniformly Bohr almost periodic in $t$. If there exists a positively bounded uniformly stable solution $\varphi(x, g, t)$, then the $\omega$-limit set $M=\Omega_{(x, t)}$ is a 1-cover of $Y$ and $M$ is an almost periodic minimal set.

This result is interesting because we are able to drop the asymptotic stability assumption which Miller and Yoshizawa used in their theories. 


\section{BIBLIOGRAPHY}

1. L. Amerio, Soluzioni quasi-periodiche, o limitate, di sistemi differenziali non lineari quasi-periodiche, o limitate, Ann. Mat. Pura Appl. (4) 39 (1955), 97-119. MR 18, 128.

2. R. Ellis, Lectures on topological dynamics, Benjamin, New York, 1969. MR 42 \#2463.

3. R. K. Miller, Almost periodic differential equations as dynamical systems with applications to the existence of A.P. solutions, J. Differential Equations 1 (1965), 337-345. MR 32 \# 2690.

4. R. J. Sacker and G. R. Sell, Finite extensions of minimal transformation groups (to appear).

5. - Lifting properties in skew-product flows with applications to differential equations (to appear).

6. G. R. Sell, Nonautonomous differential equations and topological dynamics. I, II, Trans. Amer. Math. Soc. 127 (1967), 241-262, 263-283. MR 35 \# 3187a,b.

7. Lectures on topological dynamics and differential equations, Van Nostrand Reinhold, London, 1971.

8. J. Differential Equations (to appear).

9. T. Yoshizawa, Asymptotically almost periodic solutions of an almost periodic system, Funkcial. Ekvac. 12 (1969), 23-40. MR 41 \# 2132.

Department of Mathematics, University of Southern California, Los Angeles, CALIFORNIA 90007

Department of Mathematics, University of Minnesota, Minneapolis, Minnesota 55455 